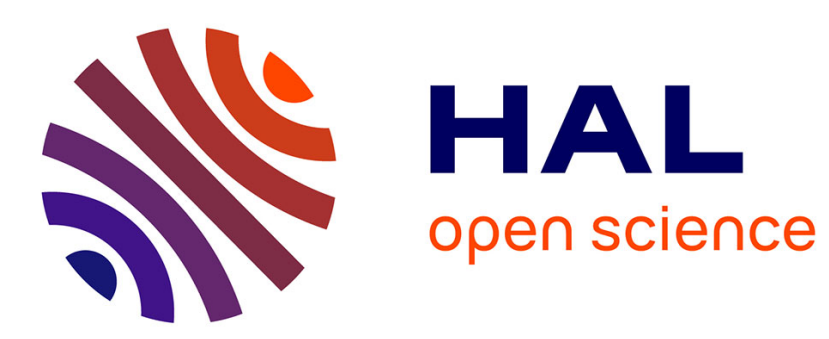

\title{
Les nouvelles routes de la soie en Méditerranée
}

Saï Belguidoum, Farida Souiah

\section{To cite this version:}

Saïd Belguidoum, Farida Souiah. Les nouvelles routes de la soie en Méditerranée. Confluences Méditerranée , 2019, 109 (2), pp.9-18. 10.3917/come.109.0009 . hal-02196580

\section{HAL Id: hal-02196580 \\ https://hal.science/hal-02196580}

Submitted on 29 Jul 2019

HAL is a multi-disciplinary open access archive for the deposit and dissemination of scientific research documents, whether they are published or not. The documents may come from teaching and research institutions in France or abroad, or from public or private research centers.
L'archive ouverte pluridisciplinaire HAL, est destinée au dépôt et à la diffusion de documents scientifiques de niveau recherche, publiés ou non, émanant des établissements d'enseignement et de recherche français ou étrangers, des laboratoires publics ou privés. 


\section{«Les nouvelles routes de la soie en Méditerranée »}

Saïd Belguidoum, Aix-Marseille Univ., CNRS, Iremam,

Farida Souiah, Aix-Marseille Univ., CNRS, Lames, LabexMed

Cet article introduit un numéro de Confluences Méditerranée consacré à la Chine en Méditerranée.

Références bibliographiques :

Saïd Belguidoum et Farida Souiah, «Les nouvelles routes de la soie en Méditerranée ». Confluences Méditerranée, 109(2), 2019, pp. 9-18. 


\section{Saïd Belguidoum et Farida Souiah}

Saïd Belguidoum, Aix-Marseille Univ., CNRS, IREMAM.

Farida Souiah, Aix-Marseille Univ., CNRS, LAMES, LabexMed.

\section{Les nouvelles routes de la soie en Méditerranée}

Initié à l'automne 2013 par le Président chinois Xi Jinping, le mégaprojet OBOR ("One belt, One road"), devenu la BRI (Belt and Road Initiative) avec pour objectif la promotion des relations transcontinentales à une ampleur inédite, suscite bien des attentions et des spéculations. S'il est impressionnant par sa dimension, les dynamiques qu'il implique ne sont pourtant pas forcément nouvelles. La renaissance des routes de la soie était déjà avancée comme hypothèse quelques années auparavant par des économistes1. Elle se traduisait par de multiples initiatives, souvent discrètes, mais efficaces, développées en grande partie par des acteurs du bas, et ce depuis l'adhésion de la Chine à l'OMC en 2001. Avec l'annonce de la BRI, la présence de la Chine dans le monde prend un nouvel essor. Ce qui était une hypothèse se confirme : les routes de la soie renaissent.

Dout laisse à croire qu'en voulant institutionnaliser son déploiement à travers le monde et en Méditerranée, la

- Chine ne cherche pas seulement à renforcer son dispositif commercial, mais à donner une nouvelle dimension à sa présence. L'expression «nowvelle(s) route(s) de la soie» désigne aujourd'hui un déploiement à partir de la Chine d'un ensemble multiforme d'initiatives économiques, commerciales, géopolitiques et diplomatiques et 
de circulations migratoires. Ces nouvelles routes suscitent fantasmes et inquiétudes, notamment lorsqu'elles se déploient en Méditerranée. La politique chinoise est alors souvent analysée à l'aune de l'émergence d'un nouvel acteur, inhabituel dans la région, faisant preuve d'activisme. Les grands chantiers ainsi que les rachats de ports, de foncier, de vignobles, d'entreprises font couler beaucoup d'encre et soulèvent de nombreuses interrogations.

La Méditerranée est un des centres mondiaux où s'effectue la reconfiguration des échanges internationaux issus de la globalisation de l'économie. Il n'est donc pas étonnant que là, comme ailleurs, les nouvelles routes de la soie se déploient. Certes la présence chinoise en Méditerranée était antérieure à l'initiative de 2013. Elle est d'ailleurs devenue visible, depuis l'adhésion du pays à l'OMC en 2001, avec le boom du Made in Cbina et l'exportation massive de produits bon marché. Mais à ces flux commerciaux se sont rajoutés progressivement des investissements de plus en plus nombreux, des rachats d'infrastructures et de nouvelles formes de circulation migratoires : celles des hommes d'affaires, mais aussi de la main-d'œuvre que les entreprises de construction chinoises suscitent lorsqu'elles mènent des grands chantiers dans différents pays de la région. Ce sont aussi les flux de touristes, de plus en plus nombreux, qui visitent les pays desservis par les nouvelles routes de la soie en Méditerranée.

Les articles réunis dans ce dossier étudient à différentes échelles les modalités différenciées de la présence chinoise sur les deux rives de Méditerranée. Il s'agit d'identifier et de comprendre tant les initiatives qui s'inscrivent dans le cadre d'une mondialisation par le bas (ou la mondialisation discrète), que celles qui viennent du haut et les velléités d'institutionnalisation des routes de la soie. D'un côté le projet BRI, qui repose essentiellement sur la puissance de l'appareil d'État chinois, de l'autre, les itinéraires commerciaux et les places marchandes constituées par les acteurs «du bas», entrepreneurs transnationaux circulant entre les différentes places discrètes de ce type de commerce qui se sont particulièrement développées depuis deux décennies. Il s'agit non seulement d'articuler les dynamiques commerciales et territoriales à diverses échelles, mais aussi de saisir les répercussions économiques, géographiques ou sociales potentielles de ces nouvelles routes de la soie.

En guise d'introduction, explorons quelques-uns des traits saillants que les articles qui constituent ce dossier permettent de dessiner, à commencer par les liens entre mondialisation par le haut et mondialisation par le bas. 


\section{Mondialisation discrète et mondialisation par le haut : les formes de la présence chinoise}

Depuis la fin des années 1990, dans le sillage de la globalisation de l'économie, s'est mis en place un commerce transnational, discret, mais efficace faisant apparaitre de nouvelles formes de l'entrepreneuriat marchand. Ce commerce transnational est lié à l'essor d'un marché de consommation des pays du Sud que les firmes multinationales ont délaissé $e^{2}$ La Chine est rapidement devenue la source principale de ces entrepreneurs marchands venant du monde entier et donc aussi des différents pays méditerranéens. Alejandro Portes, l'inventeur de la notion de mondialisation par le bas ("globalization from below»"), et Alain Tarrius ont été parmi les premiers à mettre en évidence les spécificités de l'économie transnationale. Tarrius décrit et analyse à la fin des années 1990 «la genèse, en Méditerranée occidentale, de véritables réseaux d'entrepreneurs (...) qui atteste bel et bien de l'existence d'une autre mondialisation : une mondialisation discrète, qui s'accomplit par le bas »".

C'est en décrivant ce type d'économie que l'expression de route de la soie a été employée par la recherche pour désigner l'émergence de réseaux associés au commerce transnational dont les ramifications relient différentes places marchandes dans le monde. Ces travaux, qui s'intéressent en particulier aux connexions entre le monde arabe et la Chine, mettent en lumière le rôle des entrepreneurs-migrants dans la construction de cette nouvelle route de la soie dont la vitalité ne dépend ni des gouvernements ni des entreprises, mais des individus. Dans ce numéro, la contribution de Saïd Belguidoum et Olivier Pliez, ainsi que celle de Jean-Pierre Taing donnent à voir en priorité cette mondialisation discrète.

L'article de Saïd Belguidoum et Olivier Pliez remonte les routes de la soie, mises en œuvre par les commerçants algériens depuis près de 40 ans, jusqu'à Yiwu. Cette mondialisation repose dans la mise en connexion d'espaces urbains liés entre eux par des flux de commerçants et de marchandises. Avec la mise en œuvre de la BRI on peut se questionner sur la manière dont s'établiront ou pas des liens entre les stratégies institutionnelles et celles des acteurs déjà inscrits dans les réseaux transnationaux. Le risque est que les initiatives émanant du haut entravent, plus qu'elles n'aident, ce qui est déjà construit et qui fonctionne, souvent aux marges de l'action des États. Les routes de la soie institutionnelles qu'incarnent des projets comme la BRI pourraient absorber cette économie et par là même la faire disparaitre, se 
privant ainsi de l'une des forces essentielles de la mondialisation par le bas : la labilité et la grande souplesse des réseaux qui l'animent.

Jean Pierre Taing nous propose, à partir du cas marocain, une lecture de la mondialisation des flux migratoires chinois qui accompagne l'ascension économique de la Chine sur la scène internationale. Il s'intéresse à deux types de circulation, celle des touristes et celle des commerçants apportant un éclairage sur d'autres aspects des routes de la soie : la manière dont elles sont façonnées et façonnent à leur tour les nouveaux espaces de mobilité des Chinois.

L'essor du tourisme chinois n'est pas limité au Maroc. Visible depuis plusieurs années en France et en Europe, le phénomène le devient également dans les pays du pourtour sud de la Méditerranée, notamment au Maroc comme cela fut mentionné, mais également, en Tunisie et en Égypte, pays qui étaient jusqu'alors des destinations pour les touristes occidentaux qui les ont partiellement délaissées à la suite des "printemps arabes». Les circulations ne sont pas uniquement le fait des touristes ou des entrepreneurs marchands, elles concernent également les techniciens et ingénieurs dans le cadre d'accord de coopération et les travailleurs du BTP qui s'expatrient le temps d'un chantier dans un pays étranger. C'est l'une des dimensions qu'évoque Yahia Zoubir, dans son article consacré aux relations entre la Chine et le Maghreb, et plus particulièrement en Algérie. Ces circulations s'inscrivent dans la mise en œuvre des routes et sont encouragées par la Chine qui n'hésite plus à signer des accords en faveur de la libre circulation de ses ressortissants. L'essor du tourisme chinois le long des nouvelles routes de la soie n'est donc pas une coïncidence.

Ainsi, mondialisation par le haut et mondialisation par le bas peuvent se conjuguer, se renforcer et parfois même se confondent comme le démontre avec beaucoup de justesse et de profondeur l'article d'Emile Tran. S'intéressant à Marseille, cette contribution repose sur deux études de cas: le projet du MIF 68 (Marseille International Fashion Center), vaste zone de vente de gros et semi-gros regroupant plusieurs centaines d'importateurs, emblématique de la mondialisation par le bas, mais relayée et appuyée par le haut; et le projet d'implantation de l'usine de pneus Quechen, fruit de la mondialisation par le haut alors même qu'il est le fait d'une entreprise privée. Cette contribution donne également à voir une véritable diplomatie économique qui s'exerce à renfort de visites de haut niveau et de cérémonies tant à l'échelle locale qu'à l'échelle nationale. 


\section{Les routes de la soie : des réseaux de places}

Les articles réunis dans ce dossier ne se limitent pas seulement à une étude des acteurs (individus, entreprises, États, collectivités territoriales, etc.) des nouvelles routes de la soie, ils permettent également d'explorer les lieux et les tracés de ces routes en Méditerranée. La BRI est en premier lieu une question d'infrastructures ferroviaires, routières et, plus encore, maritimes. Aussi, plusieurs de nos auteurs nous guident dans les ports de la Méditerranée : à Marseille avec Émilie Tran, dans Le Pirée, avec Christophe Chiclet, et dans plusieurs ports à l'échelle du bassin méditerranéen avec Paul Tourret. Les ports finissent toujours par être mentionnés, même dans les articles où ils ne figurent pas parmi les objets centraux de l'analyse. Dans ces ports, ce ne sont pas toujours les mêmes acteurs ni les mêmes modalités d'action qui caractérisent la présence chinoise : projet de construction, d'extension ou de rénovation, investissements, gestion ou acquisition partielle de terminaux, voire rachat de ports. Quoi qu'il en soit, il ne fait aucun doute qu'ils sont des lieux clé de la présence chinoise dans la région.

Ces considérations sur la place des infrastructures notamment portuaires dans les nouvelles routes de la soie nous conduisent à interroger la nature même de ces routes. L'article de Paul Tourret, consacré à la dimension maritime des routes de la soie est sans doute celui qui développe de la façon la plus extensive et explicite cette réflexion. Paul Tourret affirme ainsi que les routes ne doivent pas être appréhendées comme de simples tracés, mais comme des réseaux de places et de plateformes. Ces dernières ont d'autant plus de valeur pour la Chine qu'elles sont des portes d'entrée vers différents pays de la Méditerranée et au-delà. Cet argument entre en résonnance avec des éléments développés dans plusieurs des articles de ce dossier, notamment ceux qui visent à rendre compte des relations entre la Chine et un pays ou une région spécifique. L'Espagne est présentée comme une porte d'entrée vers l'Europe, l'Amérique latine, mais aussi de l'Afrique par Esteban Mario et Ugo Armanini; les pays du Maghreb, comme des portes d'entrée vers le «monde arabe», l'Afrique et l'Europe par Yahia Zoubir, etc. On peut trouver des analyses similaires dans les articles consacrés à l'Égypte ou aux Balkans. À cette échelle d'analyse, la tentation est forte d'affirmer la singularité et la position privilégiée de chaque pays. Pris dans leur ensemble, ces articles montrent qu'il y a une stratégie globale et que la Chine multiplie les plateformes en 
Méditerranée : Pékin ne semble pas vouloir se limiter à une seule porte d'entrée ni à une seule route, ce qui lui confère une certaine capacité d'adaptation en cas d'instabilité politique. Si les marchandises et les personnes peuvent facilement être redirigées vers d'autres routes et d'autres plateformes, il n'est pas aisé de faire de même avec les capitaux investis, les prêts accordés et les infrastructures acquises.

\section{Stratégie globale et approche bilatérale}

À bien des égards, on pourrait penser que la stratégie chinoise est pensée et opérationnalisée à l'échelle globale ou au moins régionale. La multiplication des forums de collaboration - Forum de coopération sino-africaine (depuis 2000), Forum de coopération sino-arabe (depuis 2004) ou encore le «16+1» qui réunit seize pays d'Europe centrale et orientale et la Chine (depuis 2012) - ainsi que les initiatives telles que la BRI (2013) confortent cette idée.

Il y a indéniablement une stratégie globale qui s'inscrit dans la continuité de l'ouverture et de la libéralisation économique de la Chine avec l'arrivée au pouvoir de Deng Xiaoping en 1978; et de la politique Going Out initié en 1999 qui visait à encourager les investissements chinois à l'étranger, comme l'écrit Émilie Tran. Les éléments analysés dans notre développement sur la place des infrastructures dans les nouvelles routes de la soie confortent cette conclusion. Pourtant, les auteurs qui contribuent à ce numéro le disent, chacun à sa manière, la Chine n'a pas de politique régionale homogène en Méditerranée. L'article de Jean-Marc Chaumet, où il analyse les nouvelles routes de la soie à l'aune des défis agricoles auxquels la Chine est confrontée, offre sans doute l'une des plus claires illustrations de cet argument. L'auteur montre que les pays du pourtour méditerranéen sont ciblés en fonction de leur potentiel agricole et de leur situation économique afin de permettre à la Chine d'atteindre ses multiples objectifs en termes de politique agricole. Certes comme cela a été mentionné, des forums régionaux existent et ont leur utilité relative, mais c'est à partir d'accords bilatéraux que se concrétisent les routes de la soie dans l'ensemble des espaces que les auteurs de ce numéro étudient: le Maghreb, l’Égypte, Israël, la Palestine, les Balkans et l'Espagne. Ce choix du bilatéralisme n'est pas sans conséquence.

Plusieurs contributions à ce dossier mettent en lumière l'asymétrie des relations entre la Chine et le pays ou la région étudiée. Dans 
leur article consacré à l'Espagne, Esteban Mario et Ugo Armanini montrent le caractère parfois ambivalent de la politique espagnole qui tout en développant considérablement ses relations économiques et commerciales cherche à rééquilibrer ses échanges en s'appuyant sur l'Europe et la nécessité de respecter un cadre multilatéral. Ainsi, les deux pays ont récemment promu un approfondissement de leurs relations, mais l'Espagne a refusé d'adhérer à l'initiative des nouvelles routes de la soie. Cette asymétrie est plus considérable dans le cas des relations de la Chine avec les Balkans, avec l'Égypte ou avec les pays du Maghreb.

\section{Une alternative aux puissances occidentales?}

Pourquoi développer et formaliser des relations économiques et politiques avec la Chine alors qu'elles sont caractérisées par une telle asymétrie? Les contributions réunies dans ce numéro apportent des éléments de réponse à cette question. La politique étrangère de la Chine post-Mao n'est pas marquée du sceau de l'idéologie. Les autorités chinoises mettent en avant des principes tels que la neutralité, la non-ingérence et les relations "gagnant-gagnant» qui séduisent de nombreux pays du bassin Méditerranéen et plus particulièrement, mais pas uniquement, ceux de la rive Sud.

Plusieurs articles mettent en lumière l'attractivité de l'offre chinoise, comparée aux propositions des puissances occidentales. Elle n'est certes pas entièrement dénuée de conditionnalité politique, comme l'illustre le caractère non-négociable du principe de «la Chine unique»", mais elle semble permettre de s'abstraire de ce qui est perçu comme un dogmatisme politique et économique des puissances occidentales. L'offre de ces dernières apparait comme d'autant moins convaincante, qu'elle repose sur un équilibre précaire entre realpolitik et valeurs prônées comme l'arguent Elena Aoun et Thierry Kellner dans leur article consacré l'extension et l'approfondissement des domaines de coopération entre Le Caire et Pékin à l'ère «Xi-al-Sissi». Les analyses développées par Yahia Zoubir sur la Chine au Maghreb font écho et confortent ces conclusions. Avec les cas du Maghreb et de l'Égypte, les auteurs mettent également en lumière la résilience de la Chine lors des crises politiques notamment grâce au principe de neutralité. Soulignons tout de même qu'accroître sa coopération avec la Chine n'est pas synonyme d'un amoindrisse- 
ment de la coopération avec les pays occidentaux, mais un effort afin de contribuer à l'émergence d'un ordre international multipolaire.

Les principes de non-ingérence et de neutralité séduisent aussi sur la rive Nord de la Méditerranée. Dans son article consacré à la Chine dans les Balkans, Christophe Chiclet montre que les Chinois sont d'autant mieux accueillis que l'Union européenne et la Russie ont déçu une région notamment affectée par la crise financière de 2008 et les contreparties imposées à la Grèce par la Troïka (FMI, BCE, et Commission européenne).

La manière dont la Chine met en œuvre le principe "gagnantgagnant» est également manifeste dans plusieurs contributions. Par exemple, lorsque Jean-Marc Chaumet analyse la politique agricole chinoise en Méditerranée, il démontre que le commerce et la coopération se conjuguent, et ce particulièrement dans les pays de la rive Sud et Est. La Chine aide et investit pour permettre à ces pays d'accroître leurs productions agricoles, pour des raisons tant économiques (faire baisser les prix sur les marchés internationaux) que politiques (améliorer son image et entretenir ses relations avec les États, notamment lorsqu'il y a des échanges dans le domaine de l'énergie). Lorsque Paul Tourret, sur les dimensions maritimes des nouvelles routes de la soie, indique que celles-ci sont l'occasion pour des pays qui en étaient jusqu'alors privés de se doter d'infrastructures portuaires et ferroviaires modernes.

Il faut souligner de nouveau que ce que la Chine a à offrir séduit également dans la rive Nord. La lutte des places et de ports afin d'accueillir les investisseurs et les flux de marchandises, mais aussi les équipements en témoignent. Dans l'article d'Emile Tran, les moyens déployés par Marseille pour accueillir le projet Quechen sont à ce titre éloquents, mobilisant jusqu'au plus haut niveau de l'État français. Ainsi, la rencontre des intérêts est sans doute le premier argument d'attractivité de la Chine.

Pourtant, le " gagnant-gagnant » a ses limites. D’une part, les grands travaux d'infrastructures ont un coût et certains pays s'engagent dans un processus d'endettement. L'Algérie est un exemple éloquent où les grands travaux ont considérablement grevé les réserves financières de ce pays (surcout de l'autoroute Est-Ouest, la grande mosquée d'Alger...). D'autre part, la question de l'asymétrie des échanges, déjà évoquée, et le déficit des balances commerciales des pays de la Méditerranée vis-à-vis de la Chine qui en découle n'est toujours pas compensé. 


\section{De l'économie à la géopolitique}

Bien que les succès remportés par la Chine en Méditerranée soient liés à sa capacité à faire des offres qui mettent en avant le principe "gagnant-gagnant», sa présence suscite tout de même de la méfiance, et est parfois analysée comme une forme de néo-colonialisme ou comme un chemin pavé vers l'émergence de la Chine comme superpuissance.

La principale source de la puissance de la Chine repose sur ses capacités économiques. Lorsqu'il est mentionné, le soft-power de la Chine est toujours qualifié de limité par les auteurs qui ont contribué à ce numéro. La capacité de la Chine à traduire son pouvoir économique en hard-power diplomatique et stratégique est également remise en question dans les contributions de ce numéro. Dans sa réflexion sur la stratégie maritime de la Chine, Paul Tourret concède qu'il existe une diplomatie de l'infrastructure et que la Chine "construit des proximités stratégiques, des amitiés ou de la 'clientèle', au point où il est possible d'affirmer qu'une diplomatie de l'infrastructure est à l'auvre». Pourtant cela reste, selon l'auteur, d'une utilité limitée pour construire un hard-power.

Le rôle de la Chine sur la scène géopolitique internationale demeure également limité. Dans un article consacré au conflit israélo-palestinien, Guy Burton argue que la position de la Chine est marquée par l'inertie et la capacité à se départir de la grille de lecture initié par les accords d'Oslo. La Chine semble réticente à traduire son influence économique grandissante avec les deux parties du conflit (bien que les relations avec Israël soient bien plus importantes) en influence politique pour aider dans la résolution du conflit.

Certains des articles nous invitent à questionner cette politique de neutralité de la Chine qui jusqu'à présent a servi ses intérêts mais qui à terme peut devenir difficile à pratiquer. Cela concerne aussi bien les conflits régionaux (Israël -Palestine, et Sahara occidental) que son attitude vis-à-vis des régimes en place. Il est légitime de se demander si cette "neutralité » ne va pas finir par "beurter les sentiments des sociétés civiles maghrébines» comme l'écrit Yahia Zoubir.

Bien que les auteurs qui ont contribué à ce numéro adoptent des échelles d'analyse variées (du local au régional) et s'emparent de thématiques et d'espace divers, leurs études entrent en résonance les unes avec les autres. Des traits saillants qui permettent de mieux 
appréhender la présence chinoise en Méditerranée émergent : les liens entre mondialisation par le haut et mondialisation par le bas, le rôle des places et des routes (tant maritimes que terrestres), la centralité des relations bilatérales pour mettre en œuvre une initiative pourtant globale, ou encore les lignes de démarcation, pas toujours claires, entre ce qui relève de l'économique et ce qui relève de la géopolitique. Au-delà de ces traits saillants, la lecture de ces articles offre une immersion dans des enjeux fondamentaux (l'agriculture, la mondialisation, les équilibres internationaux) et jouissent de la richesse et de la diversité des études de cas développés.

Notes

1. Harry G Broadman, AfricasSilk Road: China and Indias New Economic Frontier Washington DC, World Bank, 2007, 391 p; Ben Simpfendorfer, The New Silk Road. How a Rising Arab World is Turning Away from the West and Rediscovering China, Basingstoke, Palgrave Macmillan, 2009, 202 p.

2. Armelle Choplin et Olivier Pliez, La mondialisation des pauvres, Paris, Seuil, 2018, $128 \mathrm{p}$.

3. Alejandro Portes, «La mondialisation par le bas, l'émergence des communautés transnationales », Actes de la recherche en sciences sociales, n² 129, 1999, pp. 15-25. 4. Alain Tarrius, La mondialisation par le bas. Les nouveaux nomades de l'économie souterraine, Balland, 202, $169 \mathrm{p}$.

5. Selon ce principe, il n'y qu'une seule Chine dont font partis Hong Kong, Macao, Taïwan, le Tibet et le Xinjiang. 